\title{
TWO CASES OF ORTHOPTIC INTEREST
}

\author{
BY \\ E. B. Alabaster, C. Rudd and M. TŔee \\ FROM THE BIRMINGHAM AND MIDLAND EYE HOSPITAL
}

THE utility of modern orthoptic examinations in diagnosis is undoubtedly beyond dispute. The position of orthoptic treatment in our armamentarium of ocular therapeutics must, however, be continually subject to critical survey in the light of experience. This continuous readjustment of outlook necessarily obtains in all fields of therapeutic endeavour, and is especially called for in the case of recent methods in order to produce a practical balance, between "enthusiasms, possibilities, and cold facts. For this reason we feel that the details of the following two orthoptic cases are worthy of publication, and also because we are inclined to believe that more adequate use should be made, than heretofore, of the data obtainable from orthoptic examinations in the diagnostic sphere.

The first case is one with a small degree of convergent strabismus which had prolonged orthoptic treatment and whose history reveals considerations of interest. Master A. G. F. L. first attended this Hospital at the age of 2 years on March 11, 1931, and was seen by Mr. Rudd who noted a left convergent strabismus and ordered atropine for later refraction. On March 18, 1931, the following correction was ordered :-

$$
\begin{aligned}
& \text { Right. }+2 \cdot 0 \mathrm{sph} \text {. } \\
& +10 \text { cyl. axis } 170^{\circ} \\
& \text { Left. } \quad+2.50 \mathrm{sph} \text {. } \\
& +1.50 \text { cyl. axis } 165^{\circ}
\end{aligned}
$$

The next attendance was on February 27, 1935, at the age of 6 years and the patient was referred by Mr. Rudd for orthoptic training.

Orthoptic report. Right vision $=6 / 12$; with glasses $=6 / 9$. Left vision $=6 / 18$; with glasses $=6 / 12$.

Angle of Deviation. $+12^{\circ}$ without glasses and $+6^{\circ}$ with glasses. The left eye tends to slip in to about $25^{\circ}$ accommodative type of squint.

The patient attended the Orthoptic Department continuously from February 27, 1935, till November 24, 1937, except for an interval between September 24, 1936, till November 19, 1936, when he was away with appendicitis. In all he received 197 treatments, each of 20 minutes duration. The treatment included wearing an occluder on the right lens early in 1935 ; later in the "stripping" of the right lens; and exercises to combat left eye suppression. In spite of this the condition showed only slight change. The refraction was repeated on November 25, 1936, and the correction ordered gave the following visual results :- 


$$
\begin{aligned}
& \text { Right vision, with } \frac{+1.50 \mathrm{sph} .}{+0.75 \mathrm{cyl} \text {. axis } 10^{\circ}}=6 / 6 \\
& \text { Left vision; with }+1.25 \mathrm{sph} . \\
&+0.50 \mathrm{cyl} \text {. axis } 105^{\circ}
\end{aligned}
$$

On November 25, 1937, the orthoptic report was:-

$$
\begin{array}{ll}
\text { Right vision }=6 / 9 . & \text { With glasses }=6 / 6 . \\
\text { Left vision }=6 / 6 . & \text { With glasses }=6 / 6 .
\end{array}
$$

Deviation $7^{\circ}$ without glasses and $4^{\circ}$ with glasses. Fusion at $+5^{\circ}$ and abduction to $0^{\circ}$. The duction was regarded as poor, and insufficient to enable stereoscopic vision to be maintained; and as the fusion was unsteady with a tendency to left suppression, in spite of the long course of orthoptic treatment received, it was decided that further orthoptic exercises should be discontinued and the case was discharged as a cosmetic result. However, the patient attended twice in January 1938.

There was no further attendance at the Orthoptic Department from January 22, 1938, till December, 1941, when he came up to the out-patients because he had broken his spectacles. On December 3, 1941, refracted under H. and C. and on December 6, 1941, P.M.T. gave :-

$$
\begin{aligned}
& \text { Right vision, with }+0.50 \mathrm{sph} . \\
& +1.0 \text { cyl. axis } 10^{\circ}=6 / 6 . \\
& \text { Left vision, with }+0.50 \text { cyl. axis } 80^{\circ}=6 / 6 \text { ordered. }
\end{aligned}
$$

The patient was seen by Mr. Tree who asked for an Orthoptic report.

Orthoptic report.-Maddox rod fixing with right eye gave $10^{\Delta}$ esophoria. Maddox rod fixing with left eye gave $4^{\Delta}$ esophoria. Maddox wing test $=11^{\Delta} \cdot 15^{\Delta}$ esophoria ; Worth's 4 lights. Simultaneous macular perception with fusion (S.P.2) at $0^{\circ}$ with no hyperphoria. Ductions fair $\left(-5^{\circ}\right.$ to $\left.+25^{\circ}\right)$ full stereoscopic vision, that is full binocular vision with esophoria, but no symptoms.

On April 14, 1942, examination with the Maddox wing test showed a further decrease of the esophoria, i.e. $5^{\Delta}$ esophoria without glasses and $1^{\Delta}$ esophoria with glasses and no hyperphoria. The cover test, however, showed the presence of movement. Each eye moved outwards to take up fixation on removing the cover. This was more marked on uncovering the left eye. In addition the movement was less in each eye when glasses were worn.

The patient is alert and intelligent, and his mother states that he has been very co-operative throughout all his treatment. He is now 13 years of age.

His mother also states that when he was discharged in November, 1937, she noticed that when he removed his spectacles in the evenings preparatory to going to bed, his left eye would turn in, but during the course of the last few years this tendency disappeared. 


\section{Discussion}

It is difficult to be dogmatic about the effect of orthoptic exercises in this case. Whether or not the ultimate improvement after discharge was related to the treatment previously received, or whether such improvement was entirely dependent, on the natural decrease of hypermetropia, we leave open to discussion. In this connection it is to be regretted that the earlier orthoptic reports were not as full as the more recent:" "However, we think it best to allow the mere facts recorded to speak for themselves.

Here, therefore, is a case with a small degree of convergent strabismus $\left(6^{\circ}-12^{\circ}\right)$ of early onset (2 years), which commenced orthoptic treatment at the age of 6 years, with good vision in both eyes, and attended for 197 treatments during a period of 2 years and 9 months, and at the end of that time was discharged with a small degree of squint still present $\left(4^{\circ}-7^{\circ}\right)$ with poor ductions and unsteady fusion, and to be regarded merely as a cosmetic result. The patient received no further treatment for 4 years excep't for 'wearing his spectacles, and then returned at the age of 13 years with no squint present, and with full stereoscopic vision, but with an esophoria remaining which is causing no symptoms.

The second 'case provides a striking example of the wide adaptability possible in the co-ordinated use of both eyes. Considerations

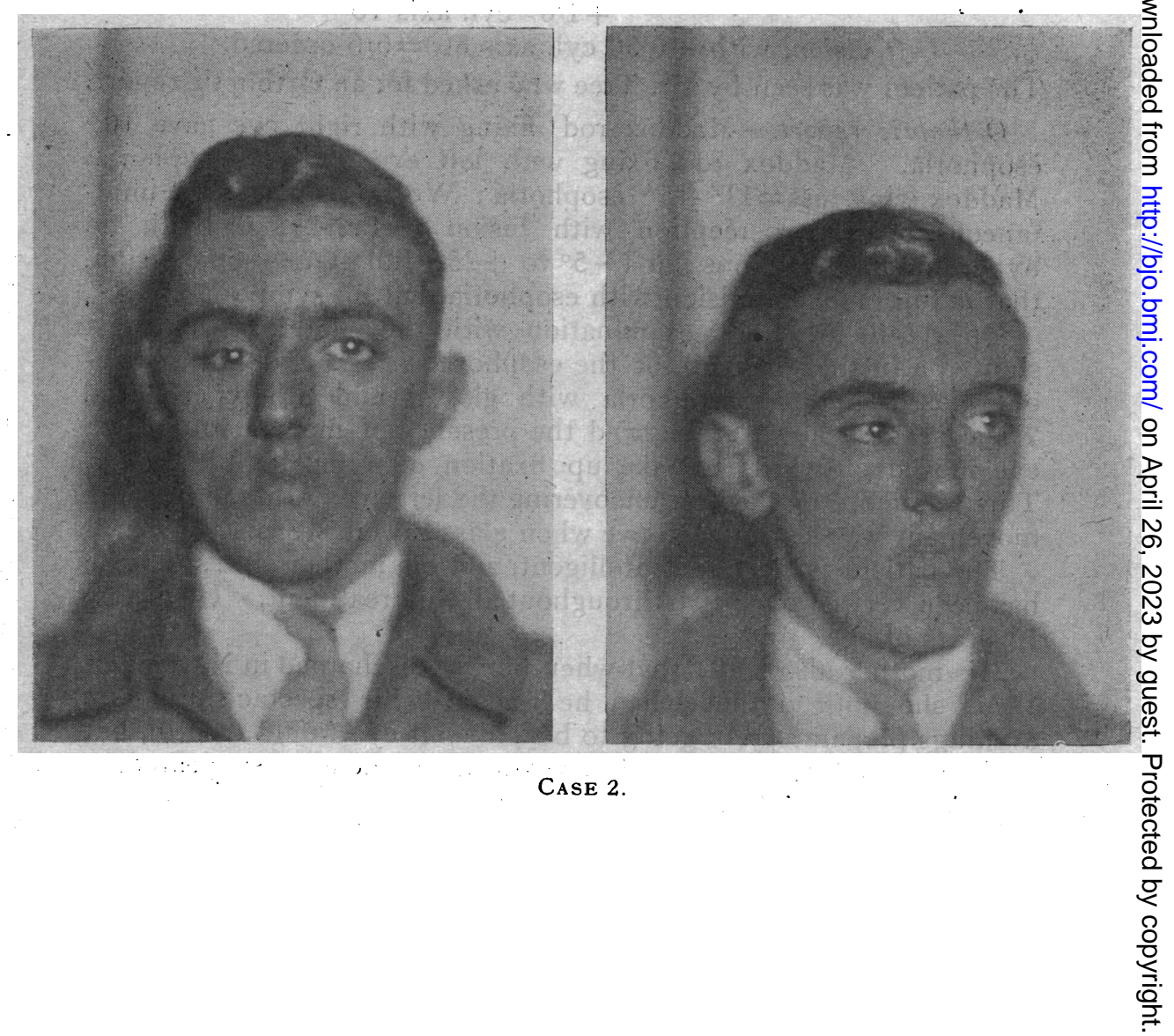


of the vast incidence of asthenopia, and the stated delicacy of the fusion faculty would lead us to believe that such adaptability is low, but here we present a case whose gross displacement of one eye to the extent of 1 centimetre following a motor accident, was followed by the retention of stereoscopic vision, with the entire absence of discomfort of any kind.

The patient, Mr. A. A., first attended here under the care of Mr. Alabaster on May 8, 1941, when he was $18 \frac{1}{2}$ years of age. He stated that he had been involved in a motor car accident on March 21, 1941, was ? unconscious for a short time and was admitted to the Birmingham General Hospital suffering from a fractured skull, retained for 2 weeks, and then transferred to the Stafford Emergency Hospital for 4 weeks. He had had an incised wound over the right eyebrow and this had been sutured. He had noticed diplopia for 2 days only after being unbandaged, and there had been no recurrence since: He stated that he had come to the Eye Hospital because he was worried by the marked downward displacement of his right eye, and because he had noticed that on closing the left eye objects seemed to be very far a way.

On examination the photographs reveal something of the condition present :-

Right vision $=6 / 18$; with $+0.50 \mathrm{sph} .=6 / 12$.

Left vision $=6 / 6$.

Oblique scar present over the right eyebrow, the right palpebral aperture is considerably lower than the left, so that the downward

-

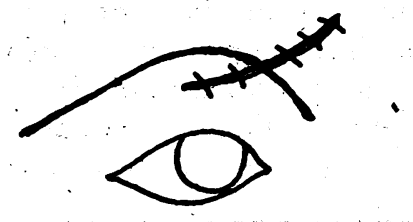

displacement of the right eye is about 1 centimetre. There is also enophthalmos of the right eye so that measurements with Hertel's exophthalmometer showed the apex of the right cornea at $16 \mathrm{~mm}$., and the left cornea at $20 \mathrm{~mm}$. from the orbital margin. There is flattening of the right side of the face, and palpation of the right orbital margins showed irregularity at the junction of the middle and inner thirds along both the upper and lower margins.

The $X$-ray films showed fracture of the right malar bone passing obliquely down and out from the orbital margin, and a fissured fracture in both the roof and floor of the right orbit passing directly backwards from the orbital margins at the sites of irregularity already noted on palpation. The right antrum was somewhat opaque and this was thought to be possibly due to haemorrhage. 
The first orthoptic report on May 8, 1941, stated:-Fusion at $0^{\circ}$ with ductions from $-2^{\circ}$ to $+35^{\circ}$. Good stereoscopic vision. 4 Worth's lights looking straight ahead. Maddox rod showed tendency to esophoria and slight right hyperphoria. By July 3, 1941, the patient's only complaint was that distant objects seemed to vary in size, appearing to get smaller and then enlarging. Apart from this he had no headaches and no diplopia. No head tilting was observed.

On January 29,1942 , as examination with red and green goggles revealed a tendency to diplopia in the extreme oblique peripheries of the field, but no diplopia in the horizontal plane, he was sent for more complete orthoptic examination and for a report on the field of diplopia.

Second orthoptic report. Fusion at $+4^{\circ}$ with ductions from $0^{\circ}$ to $+134^{\circ}$. Full stereoscopic vision.

Maddox rod. $1^{\Delta}$ esophoria with no hyperphoria looking ahead and fixing with either eye.

$6^{\Delta}$ esophoria with $10^{\Delta}$ right hyperphoria on looking downward and fixing with either eye.

$2^{\Delta}$ esophoria with $1^{\Delta}$ left hyperphoria on looking up and fixing with either eye.

Worth's 4 dot test. Looking ahead 4 lights. Looking down 5 lights $\mathrm{r} / 1$; looking up 5 lights $\mathrm{l} / \mathrm{r}$.

Field of diplopia revealed displacement in the extreme oblique positions, especially in the downward directions and implicating the following muscles of the right eye-the superior and inferior obliques, and the superior and inferior recti.

The patient, who is engaged on aeroplane engine maintenance, stated that he ordinarily thotices' no diplopia, drives a car to and from his work without trouble, occasionally rides a motor-cycle, and as part of his work he does aeroplane testing in flight, when he takes

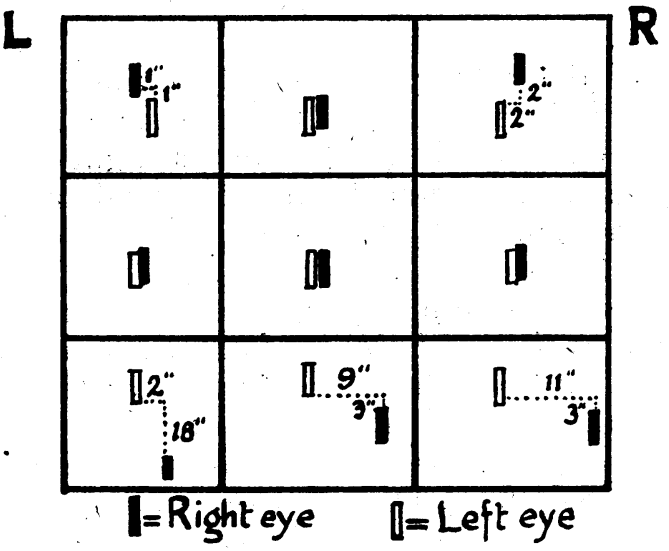


over the controls. It must be emphasised that this patient has had no orthoptic training at any time, and in spite of the great displacement of his right eye, experienced only a minor degree of diplopia as a temporary phenomenon. Since then he has been proved to possess good stereoscopic vision sufficient to have adequate control of a motor cycle, a motor car, and an aeroplane in flight. The photographs show the displacement of the right eye.

We wish to record our indebtedness to Miss Bennett and Mrs. Edwards of the Orthoptic Department for their very ready assistance and co-operation in the elucidation of these two cases. In addition we wish to thank Dr. H. Black, Radiologist to the Hospital for his opinion on the X-ray appearances in Case 2. Our thanks are also due to Mr. W. E. Meggeson for kindly photographing this patient.

\title{
THE USE OF M. AND B. 693 IN POST-OPERATIVE OCULAR SEPSIS
}

\author{
BY \\ Allister M. MacGillivray \\ DUNDEE
}

THE following case is of interest as illustrating the efficacy of M. and B. 693 in post-operative ocular sepsis.

The patient, a woman aged 52 years, was operated on for simple chronic glaucoma. The left eye was trephined, with a peripheral iridectomy, the conjunctival flap being stitched into position with a continuous silk suture. The suture was removed on the fourth day. On the morning of the seventh day, the conjunctival wound was found to be injected and oedematous. By 6 p.m. on the same day, flakes of pus could be seen adhering to the wound, and several small conjunctival haemorrhages had developed. The whole of the upper bulbar conjunctiva was injected and oedematous. Pus could be seen draining through the trephine opening into the anterior chamber. The aqueous was turbid, and the visual acuity reduced to $1 / 60$. The frequent instillation of atropine sulphate 1 per cent. and argyrol 10 per cent. was ordered, and half hourly bathings with really hot boric lotion. Two tablets of $M$. and $B$. 693 were given at 6 p.m., and repeated every four hours. This treatment was carried on throughout the night. Fortunately, in spite of intense nausea, the patient was able to retain the tablets. The following morning; the wound was found to be clear of pus. The injection and chemosis of the conjunctiva had subsided, and 